Miller, S., Ruttinger, V., Rutledge, M. M., Frahm, R., Maurer, S., Moyer, E. Z., Kaucher, M., and Macy, I. G. (1950). Human milk studies: essential amino acids in human colostrum and transitional milk. Journal of Nutrition, 40, 499-514.

Scriver, C. R., and Rosenberg, L. E. (1973). Amino Acid Metabolism and its Disorders, p. 320. Saunders, Philadelphia.

David Burman,* John Holton, and John Allen Bristol Royal Hospital for Sick Children and Department of Clinical Chemistry, Southmead Hospital, Bristol.

*Correspondence to Dr D. Burman, Bristol Royal Hospital for Sick Children, St. Michael's Hill, Bristol BS2 8BJ.

\section{Conservative treatment of a BCG osteomyelitis of the femur}

BCG osteomyelitis and arthritis after BCG vaccination are rare considering the large number of newborns vaccinated (Foucard and Hjelmstedt, 1971). An unequivocal diagnosis can be obtained only by biopsy and bacteriological and histological examination (Paisseau et al., 1941; Backman and Wallgren, 1954; Felländer, 1963; Foucard and Hjelmstedt, 1971). The case presented here was diagnosed on clinical and radiographical criteria. Conservative tuberculostatic treatment was successfully applied. No surgical treatment was necessary.

\section{Case report}

The patient, a 3-year-old boy born to healthy parents after a normal pregnancy and birth, was vaccinated with BCG intracutaneously in the neonatal period without adverse reaction. He had never been seriously sick and had no known tuberculous contact in his family. 2 months before admission to hospital he slightly injured his right knee, after which he complained of pain in the right thigh and started to limp. Total blood counts and ESR at that time were normal. Antibiotics were given, but 6 weeks after the trauma slight erythema of the right knee appeared with local swelling and tenderness but no impairment of movement.

On examination at our clinic a number of enlarged though not tender lymph nodes were noted in the groin in addition to the above findings. Temperature was $37.4^{\circ} \mathrm{C}$ and ESR $15 \mathrm{~mm}$ in one hour. Radiographs of the right knee (Fig. 1) showed a round, localized osteolytic lesion in the metaphysis of the

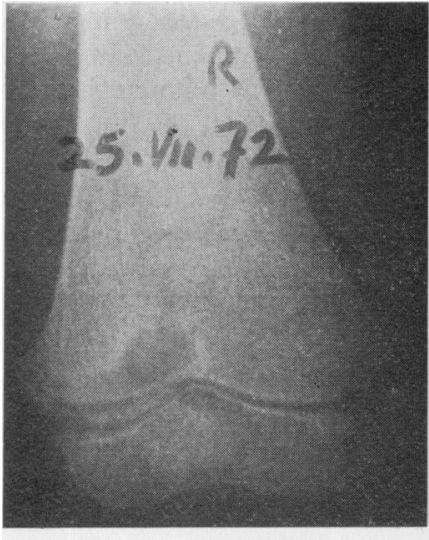

(a)

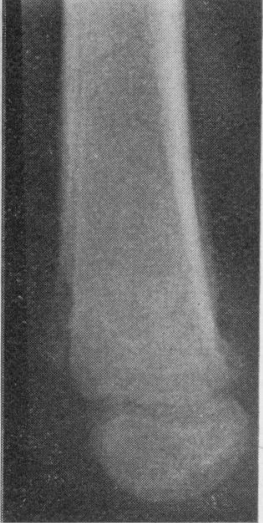

(b)
Fig. 1 (a) Osteolytic lesion in right lateral femoral condyle bordering epiphyseal plate. (b) Note periosteal reaction.

femur, bordering the distal epiphyseal plate, with a periosteal reaction in the supracondylar area. Treatment consisted of immobilization and large doses of antibiotics. 2 weeks later neither clinical nor radiological improvement was evident. At this time the ESR was $20-30 \mathrm{~mm}$ in one hour, $\mathrm{Hb} 14 \cdot 1$ $\mathrm{g} / \mathrm{dl}$, WBC $6 \cdot 8 \times 10^{9} / 1\left(6800 / \mathrm{mm}^{3}\right)(2 \mathrm{stab}, 61$ polynuclears, 33 lymphocytes, 1 eosinophil, 3 mononuclears), blood glucose $4.5 \mathrm{mmol} / 1(82 \mathrm{mg} / 100 \mathrm{ml})$, and urea $3.8 \mathrm{mmol} / \mathrm{l}(23 \mathrm{mg} / 100 \mathrm{ml})$. Mantoux test was positive $1: 1000$. $X$-ray examination of the right knee showed no further progression. A chest and skeletal survey were negative with no evidence of other foci.

The lesion seemed to be an infection of low virulence. Since there was no regression after massive antibiotic treatment (effective against Gram-positive cocci) and fewer than 4 years had elapsed since the BCG vaccination, BCG osteomyelitis of the femur was suspected. Because of the slow progression it was decided to carry out a trial treatment with tuberculostatic drugs alone. Sodium paraaminosalicylic acid $2 \mathrm{~g} / \mathrm{d}$, isoniazide $75 \mathrm{mg} / \mathrm{d}$, and streptomycin intramuscularly twice weekly were given for a period of 2 months. 5 weeks after treatment began the boy still had a slight limp but did not complain of pain. $X$-ray examination showed that the periosteal reaction had disappeared (Fig. 2). A month later the limp had also gone and treatment was stopped. When last seen $3 \frac{1}{2}$ years later he had no symptoms or signs. A radiograph of the femur showed a normal bone structure, no periosteal reaction, and the epiphyseal plate undamaged. 


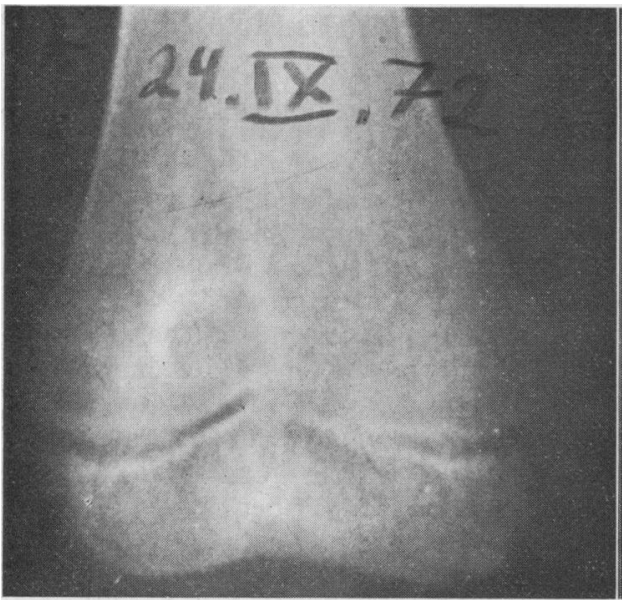

(a)

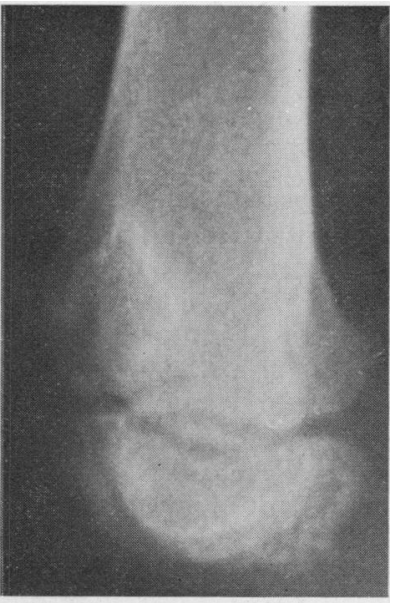

(b)
Fig. 2 Five weeks after tuberculostatic treatment. (a) Lesion appears unchanged, but $(b)$ periosteal reaction has disappeared.

\section{Discussion}

Pauker et al. (1972) described 2 cases of BCG osteomyelitis and osteoarthritis of the knee joint with positive pathologico-anatomical findings but negative bacteriological findings. In the present case the infection seemed to be of low virulence, no regression was seen after several weeks' treatment with antibiotics effective against Gram-positive cocci, and fewer than 4 years had elapsed since the BCG vaccination. In accordance with the criteria of Foucard and Hjelmstedt (1971) we therefore concluded the lesion was probably BCG osteomyelitis. Since our experience and that of others (Paisseau et al., 1941 ; Backman and Wallgren, 1954; Felländer, 1963; Foucard and Hjelmstedt, 1971) was that even biopsy may not give the diagnosis with certainty we decided to try conservative treatment. The prompt healing with tuberculostatic treatment was in our opinion sufficient evidence that the diagnosis and management were correct.

On the basis of this case a 2-month-old baby girl with an osteolytic focus of the humerus near the site of the BCG inoculation was recently treated elsewhere in the same conservative way with good results.

\section{Summary}

A 3-year-old boy presented with an osteolytic lesion and periosteal reaction in the distal metaphysis of the right femur which failed to respond to immobilization and intensive antibiotic treatment. Since the infection seemed to be of low virulence and fewer than 4 years had elapsed since BCG vaccination BCG osteomyelitis was suspected. Tuberculo- static treatment led to rapid recovery and surgical measures were unnecessary.

\section{References}

Backman, A., and Wallgren, E. I. (1954). On tuberculosis of the bones and joints of BCG vaccinated children. Acta Paediatrica, 43, 252-258.

Felländer, M. (1963). Tuberculous osteitis following BCG vaccination. Acta Orthopaedica Scandinavica, 33, 116-126.

Foucard, T., and Hjelmstedt, A. (1971). BCG-osteomyelitis and -osteoarthritis as a complication following BCG vaccination. Acta Orthopaedica Scandinavica, 42, 142-151.

Marks, J., Jenkins, P. A., Kilpatrick, G. S., Engbaek, H. C., and Vergmann, B. (1971). Pulmonary tuberculosis due to BCG. British Medical Journal, 3, 229-230.

Paisseau, G., Sorrel, E., and Ngugen, K. V.(1941). Mal de Pott sous-occipital chez un nourrisson vacciné au BCG. Bulletins et Mémoires de la Societé Medicale des Hôpitaux de Paris, 57, 328-333.

Pauker, M., Lotem, M., Levy, M., and Luria, M. (1972). BCG arthritis and osteomyelitis. Harefuah, 83, 429-432.

M. Pauker, ${ }^{*}$ M. Seelenfreund, and G. Morain Department of Orthopedic Surgery and Traumatology, Beilinson Medical Center, Sackler School of Medicine, Tel Aviv University, Petah Tikva, Israel.

*Correspondence to Dr M. Pauker.

\section{Symptomatic sinus arrest in a young girl}

A recent report (Scott et al., 1976) on symptomatic sinoatrial node dysfunction noted its infrequency in children and no reported occurrence of the 